\title{
Exploring Fine Structures of Photoactive Yellow Protein in Solution Using Wide-Angle X-ray Scattering ${ }^{*}$
}

\author{
Tae Kyu Kim, Xiaobing Zuo, ${ }^{\ddagger}$ David M. Tiede, ${ }^{\ddagger}$ and Hyotcherl Ihee \\ Department of Chemistry and School of Molecular Science (BK21), KAIST. Daejeon 305-701. Korea \\ ${ }^{*}$ Chemistry Division, Argonne National Laboratory, Angome, Hhinois 60439. LSA \\ Received Mav 11, 2004
}

\begin{abstract}
We demonstrate that wide-angle X-ray scattering pattern from photoactive yellow protein (PYP) in solution using a high flux third generation sy'nchrotron $\mathrm{X}$-ray source reflects not only the overall structure. but also fine stnictures of the protein. X-ray scattering data from PYP in solution have been collected in $q$ ranges from 0.02 $\AA^{-1}$ to $2.8 \AA^{-1}$. These data are sensitive to the protein structure and consistent with the calculation based on known crystallographic atomic coordinates. Theoretical scattering patterns were also calculated for the intermediates during the photocycle of PYP to estimate the feasibility of time-resolved wide-angle X-ray scattering experiments on such proteins. These results demonstrate the possibility of using the wide-angle solution X-ray scattering as a quantitative monitor of photo-induced structural changes in PYP.
\end{abstract}

Key Words : Wide-angle X-ray scattering. Photoactive yellow protein. Time-resolved, Biological macromolecule, 3-Dimensional structure

\section{Introduction}

The major purposes of strictural molecular biology include identifying structural changes of biological macromolecules and correlating these changes to their biological functions. Over the past years. three-dimensional structures of vast number of biological molecules have been determined using X-ray crystallography and nuclear magnetic resonance (NMR). ${ }^{1}$ However, these high resolution methods have their own limitations and therefore can be applied only when rather specific conditions are met. For example. structural determination by $\mathrm{X}$-ray crystallography requires high-quality protein crystals whose preparation sets one of the major bottlenecks. NMR overrides this requirement and allows structures in solution to be studied, but the size of the protein typically accessible by NMR is still much smaller than that of X-ray cry stallography.

In addition, the majority of the determined 3-D structures represent the ground state of bio-molecules. While these static structures carry enormous anount of information. the structures of intermediates should be known in order to correlate the structures to their functions and to understand the detailed mechanisms. ${ }^{3}$ To this goal. X-ray crystallography has progressed to adopt time-resolved studies. and recently picosecond time-resolution using this techuique has been demonstrated ${ }^{4.7}$ With this success, time-resolved X-ray crystallography holds a great promise in its applications to determine the detailed 3-D structures of transient internediates. At this point. one important question arises among scientists in this field: whether the intermediate structures deternined by time-resolved X-ray crystallog-

\footnotetext{
Dedicated to Professor Yong Hae Kim for his distinguished achievements in organic chemistry

To whom correspondence should be addressed. e-mail: Hyotcherl.Ihee akaist.ac.kr
}

raply correctly represent the reality in solution. To answer this question and to provide a complementary tool for timeresolved studies of macromolecules, we propose to utilize wide-angle $X$-ray scattering in solution.

Solution X-ray scattering of protein ${ }^{8}$ has been widely used to determine overall structure of native particle in various physiological conditions and conformational change due to the variation of external parameters. Usually small-angle scattering region is used to deternine general structural features such as the overall size and shape. This small-angle $\mathrm{X}$-ray scattering (SAXS) teclunique ${ }^{9.10}$ has achieved considerable success in restoring three dimensional structures of proteins from the scattering patterns. However, the limited information in the relatively small $q$ region, i.e. $q \leq 0.2 \AA^{-1}$, (where $q=4 \pi \sin \theta / \lambda$ is the magnitude of scattering vector. $\theta$ is half of the scattering angle and $\lambda$ is the wavelength) is an obstacle to the capability to restore three dimensional structures. ${ }^{\text {Il }}$ Therefore the measurement of scattering profile for a variety of proteins should be extended to high $q$ region with high accuracy. It is important to detect wide-angle (high-angle and wide-angle are used interchangeably in this article) scattering patterns since it directly probes distance correlations on length scales that are small compared to the dimensions and contains rich information of detailed fine structure of protein in solution. The practical use of high $q$ region data in the solution scattering experiments is limited by the difficulty of measuring the weak protein scattering signal which is averaged out with a much stronger background signals from the solvents and solution container. However, the recent advancements of high-flux third generation synchrotron X-ray source and detectors make the measurement of wide-angle scattering patterns for proteins in solution experimentally possible. . $^{1.13}$ Recently. it was reported not only that wide-angle scattering patterns from insertion-device beamline at third generation X-ray synchro- 
tron sources are sensitive to protein conformation states, ${ }^{1+}$ but also that the measured scattering patterns can be compared quantitatively with calculated patterns from detailed structural models. ${ }^{14,15}$

If the time-resolved $\mathrm{X}$-ray wide angle scattering is realized in solution. it will have many advantages over the timeresolved X-ray crystallography. First. it does not require highly-diffracting crystals and this will widen its applicability to a variety of systems. Second, any desired conditions including near-physiological one can be easily realized. Third. irreversible reactions, which are hard to study with the current time-resolved crystallography, can be studied since a simple flow cell will provide a fresh sample for each laser shot or other perturbation.

Photoactive yellow protein (PYP) ${ }^{16}$ is a small (125. residue. $14 \mathrm{kD}$ ) blue light-sensitive protein found in the purple phototrophic bacterium Halorhodospira halophila. It has been proposed as a blue light receptor for negative phototaxis of the bacterium based on the similarity of the visible absorption profile of the protein and the action spectrum of the organism. ${ }^{17}$ Since PYP is the first example of photoreceptor protein responsible for phototropic signal transduction, it is one of the most suitable targets for studying the light-capturing mechanism of living cells at atonic level. For this reason. PYP has been extensively investigated by spectroscopy ${ }^{18}$ and X-ray crystallography. ${ }^{19.21}$ Upon excitation by blue light, the chromophore of PYP. trons p-coumaric acid. switches to the cis form. ${ }^{2}$ Eventually this returns to the original form, completing a photocycle (Figure 1). ${ }^{13.2+}$ The phototropic signal transduction pathway is not known. but the conformational change occurring on the formation of the $\mathrm{pB}$ state and its relaxation to the dark state $\mathrm{pG}$ are suggested to be responsible for relaying the signal to the next step of activation mechanism.

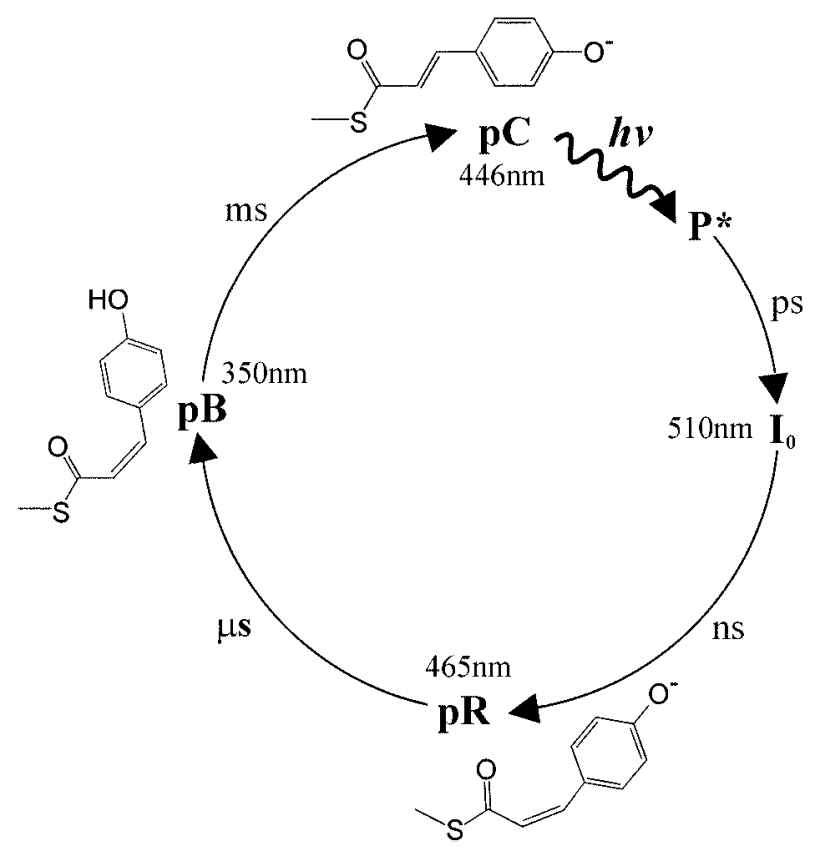

Figure 1. Photocycle of PYP in solution. pG: ground state; pR: redslifted state: $\mathrm{pB}$ : blue-shifted signal transduction state. ground state structure of PYP has been determined by X-ray crystallography ${ }^{21}$ and by solution NMR. ${ }^{21}$ However. there are noticeable differences between crystal structure of the pG state of PYP determined by X-ray crystallography and the ensemble of solution structures revealed by the NMR spectroscopy. In addition, X-ray crystallographic structures are also available for the stationary $\mathrm{pB}$ state. ${ }^{.6}$

In this work. we have carried out wide-angle solution scattering experiments of PYP. to elucidate an availability of the wide-angle synchrotron radiation $\mathrm{X}$-ray scattering. Here we demonstrate that $\mathrm{X}$-ray scattering data from proteins in solution covering the $q$ range from $0.02 \AA^{-1}$ to $2.8 \AA^{-1}$ are consistent with the calculation ${ }^{15}$ based on known crystallographic atomic coordinates and NMR structures. We show that scattering patterns in high angle region are very sensitive to fine structures of a protein in solution. We also report simulated scattering patterns from the $\mathrm{pG}$ and $\mathrm{pB}$ states of PYP for time-resolved solution X-ray scattering.

\section{Material and Method}

Sample Preparation. Apo-PYP was expressed ${ }^{19.22}$ in Escherichia coli and reconstituted ${ }^{27}$ with the cluromophore to yield holo-PYP as described in the literatures. Finally PYPs were suspended in $50 \mathrm{mM}$ phosphate $(\mathrm{pH} 7.0)$ and $20 \mathrm{mM}$ $\mathrm{NaCl}$ buffer at the concentration of $30 \mathrm{mg} / \mathrm{mL}$.

$\mathrm{X}$-ray Scattering Measurements. Wide angle scattering experiments were carried out at the undulator beam line 12ID at the Advanced Photon Source (APS). Argonne National Laboratory. The X-ray scattering instrument utilized a doublecrystal $\mathrm{Si}(111)$ monochromator and a two-dimensional mosaic $C C D$ detector for detection. ${ }^{12}$ The $X$-ray wavelength was set at $\lambda=0.685 \AA$ and the sample-to-detector distance was adjusted to measure scattering across the range of momentum transfer $0.02 \leq q \leq 2.8 \AA^{-1}$. Protein solution and buffer background two-dimensional scattering pattents were measured as the average of $10 \mathrm{X}$-ray scattering images collected at $\mathrm{I}$ sec intervals with $0.1 \mathrm{sec}$ exposure times using a $1.5 \mathrm{~mm}$ capillary flow cell and a sample flow rate of $5 \times 10^{-5} / / \mathrm{min}$. One-dimensional, azimuthally-averaged $l(q)$ scattering patterns were determined from the scattering images, and protein scattering patterns were obtained by the $\mathrm{X}$-ray transmission normalized subtraction of the buffer scattering pattern. Details of the 12ID beamline, ${ }^{28} \mathrm{X}$-ray scattering instrument. ${ }^{12}$ and protein X-ray scattering measurements ${ }^{28}$ have been previously described.

Calculation of Scattering Pattems. The general theory of solution X-ray scattering can be found in many literatures ${ }^{9.29}$ and a brief summary is given in this section. Solution scattering pattem. $I(q)$. can be represented as follows:

$$
I(q)=n P(q) S(q)
$$

where $n$ is the concentration of macromolecule in solution. $P(q)$ is the macromolecular form factor, and $S(q)$ is the solution structure factor. Here, we consider protein in dilute solution. Under this condition, we can assume that $S(q) \approx 1$ and the scattering patterns can be interpreted as form factors 
calculated from atomic coordinates. The method of calculating scattering patterns using atomic coordinates was first developed by Svergun et al. ${ }^{9}$ and was extended to produce accurate calculation of scattering patterns in high angle region using numerical precision. ${ }^{1+1 \leqslant}$ In this approach. protein scattering is calculated from average of partial scattering amplitudes from the fixed atomic coordinated in the molecules, $A_{u}$, and the solvent excluded volume, $A_{s}$

$$
P(q)=\left\langle\left|A_{a}(q)-A_{s}(q)\right|>_{\Omega}\right.
$$

where $\Omega$ represents the unit solid angle. The coherent scattering amplitude for the protein including the DebyeWaller atomic fluctuation factor ${ }^{3}$ is given by:

$$
A_{a}(q)=\rho_{0} \sum_{j=1}^{N} f_{j}(q) e^{2 q \cdot r_{j}} e^{-B_{j} q^{2}: 16 \pi^{2}}
$$

where $f_{i}$ is the atomic form factor. $B_{j}$ is the Debye-Waller Bfactor. and $N$ is the total number of non-hydrogen atoms. Hydrogen atoms. which make a significant contribution to the total protein scattering are grouped to the corresponding non-hydrogen atoms. $f_{i}$ for the group is calculated from the group atomic geometry. The Debye-Waller B-factor is proportional to the mean square position fluctuation and to the absolute temperature.

The solvent excluded volume was estimited by the dummy atom model. ${ }^{9.3(1}$ The total scattering amplitude for the dumnly aton is:

$$
A_{s}(q)=\rho_{0} \sum_{j=1}^{N} g_{j}(q) e^{i q \cdot r_{i}}
$$

where $\rho_{i}$ is solvent electron density and $g_{j}$ is the form factor for the dununy atoms with volume $V_{j}$.

$$
g_{j}(q)=G(q) V_{j} e^{-q q^{2+i 3 j+n}}
$$

and $G(q)$ is a volume expansion factor ${ }^{9}$ given as:

$$
G(q)=\frac{V_{a}}{V_{m}} e^{-q^{2}\left(r_{0}^{2}-J_{m}^{2 * 3}\right)+\pi}
$$

where $V_{j}$ is the expanded atomic volume and $V_{m}$ is the average atomic volume for the group.

Using these calculation procedures. we demonstrate the sensitivity of wide-angle scattering pattents to the details of protein structures.

\section{Results and Discussion}

Static Wide-angle Scattering Pattern on PYP in Solution. Figure 2 shows experimental and calculated scattering patterns for PYP in solution. The observed scattering pattern in the small angle region below $q \leq 0.11$ $\AA^{-1}$ shows a broad peak due to the electrostatic based interparticle interaction. For the globular particle sy stems in solution. the repulsive interparticle interaction usually produces a broad single peak in the small $q$ region. ${ }^{31}$ The

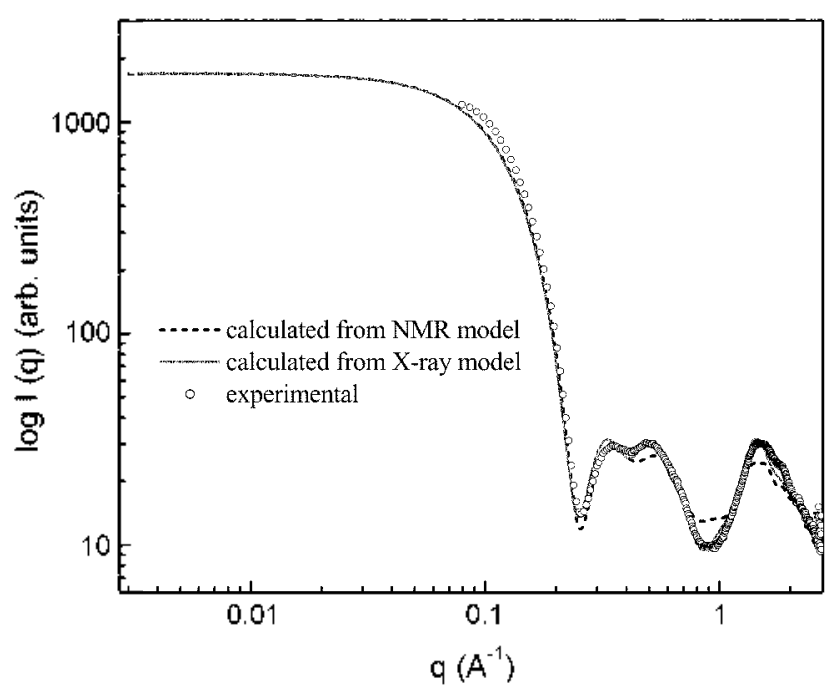

Figure 2. Comparison of experimental and calculated scattering pattems from $X$-ray and NMR model. Experimental scattering patterns were measured with protein concentration of $30 \mathrm{mg} / \mathrm{mL}$ for PYP.

amplitude and position of the broad shoulder peak depend on nonrandom particle distribution in solution. characterized by the solution structure factor. In ligh ionic strength buffers. electrostatic screening reduces the interaction, and therefore scattering profile for protein accurately reflects the protein form factor, not the solution structure factor (i.e., $I(q)$ $=n P(q) S(q)$. where $S(q) \rightarrow 1)$. The experimental scattering pattem for PYP is in good agreement with the calculated scattering pattern based on the crystal atomic coordinate in overall $q$ range. A slight deviation in snall angle region reflects interparticle interaction have not been minimized in our experiment. ${ }^{14,22}$ However. the scattering pattem in high angle region is not altered by interparticle interaction. ${ }^{14}$

There are two different structural models for PYP determined by $X$-ray crystallography ${ }^{33}$ and solution NMR spectroscopy. ${ }^{-1}$ The calculated scattering profile from the NMR model is the average of 26 similar structures. There is no Debye-Waller factor for the NMR structure in the pdb file, therefore the calculated scattering patterns from the NMR and X-ray crystallographic model were obtained without Debye-Waller effects for comparison. The scattering pattems for structures determined by these two tecluniques show significant differences at high angle regions. but they are indistinguishable at small angle. $q \leq 0.2 \AA^{-1}$. This finding can be explained by the fact that the X-ray and NMR models have similar overall size and shape. but have different fine structures. This implies that scattering pattern in high angle region is very important in depicting fine structures of protein and structural dynamics. A major factor affecting the minor discrepancy between experimental scattering pattenns and those calculated from the NMR model is the unweighted averaging of structures across the NMR ensembles. We note that scattering patterns calculated for the individual structures within this ensemble show a wide variation. with only a subset of the structures being compatible with experimental data. 

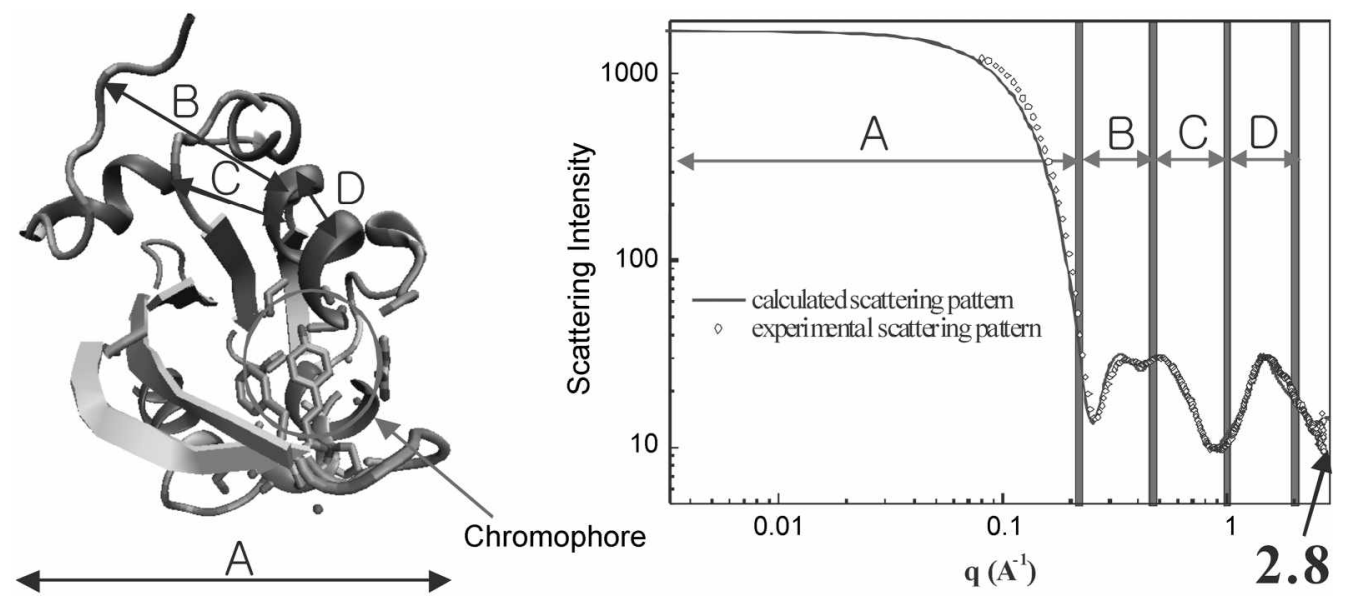

Figure 3. The regions of the experimental and calculated X-ray scattering patterns on PYP which corresponds to typical intramolecular distance in structure of PYP are shown.

The wide-angle scattering patten for PYP in solution shows noticeable oscillatory feature which is in good agreement with the calculated scattering pattern from crystal atomic coordinates. The minor disagreement at high angle region can be attributed to the fact that PY P in solution may be composed of many degrees of ensemble structures rather than one fixed and rigid structure. ${ }^{21}$ The thermal motion of a protein trapped in the crystal lattice would be more restricted than that in solution. Therefore, thermal motions of proteins in solution can be enhanced than those in crystal, which contribute more efficiently to the smearing of the experimental scattering patterns at high-angle region and this effect should be taken into account. ${ }^{14.15 .3+}$ In the simulation, the thermal effect is considered using crystallographic B-factor multiplied by a scaling factor to match the calculated and experimental high angle scattering patterns. This correction accounts for the effect due to individual incoherent atomic thermal factors. Other types of motions anticipated for proteins, uncluding the rigid-body movement of groups of atonns, can significantly alter high-angle scatterng patterns. ${ }^{14,34,35}$ It was reported that the calculated scattering pattern for horse cytochrome $\mathrm{c}$ required the $\mathrm{B}$ factor multiplied by a factor 3 to match the experimental scattering factor, ${ }^{14}$ which indicates the existence of significant configurational fluctuations for this protein in solution. However. in the case PYP, the best fit was obtained without using any B-factors. which suggests that the PYP in its pG state may be thermally very stable and compact compared with other proteins. The temperature factor affects significantly on the scattering at high angle region and has negligible effects on small angle scattering pattem, implying that scattering profiles in wideangle region are well indicative of dynamic structure of protein in solution. Therefore, wide-angle scattering patterns can be explained with the calculated patterns using atomic crystallographic coordunate provided that the thenmal effect is properly considered. ${ }^{14,34}$

Comparison of scattering patterns of other protems ${ }^{13}$ in solution with that of PYP shows that there is significant difference of shape in the $q$ range from $\sim 0.2 \AA^{-1}$ to $-2.8 \AA^{-1}$.
This suggests that wide-angle scattering patterns reflect detailed intramolecular structures of proteins and can serve as a finger print region. The scattering patterns in the $q$ range from $\sim 0.2 \AA^{-1}$ to $\sim 0.7 \AA^{-1}$ and the range from $\sim 0.7 \AA^{-1}$ to $-2.8 \AA^{-1}$ correspond to the domain structure and the secondary structure. respectively. In Figure 3. domains in the
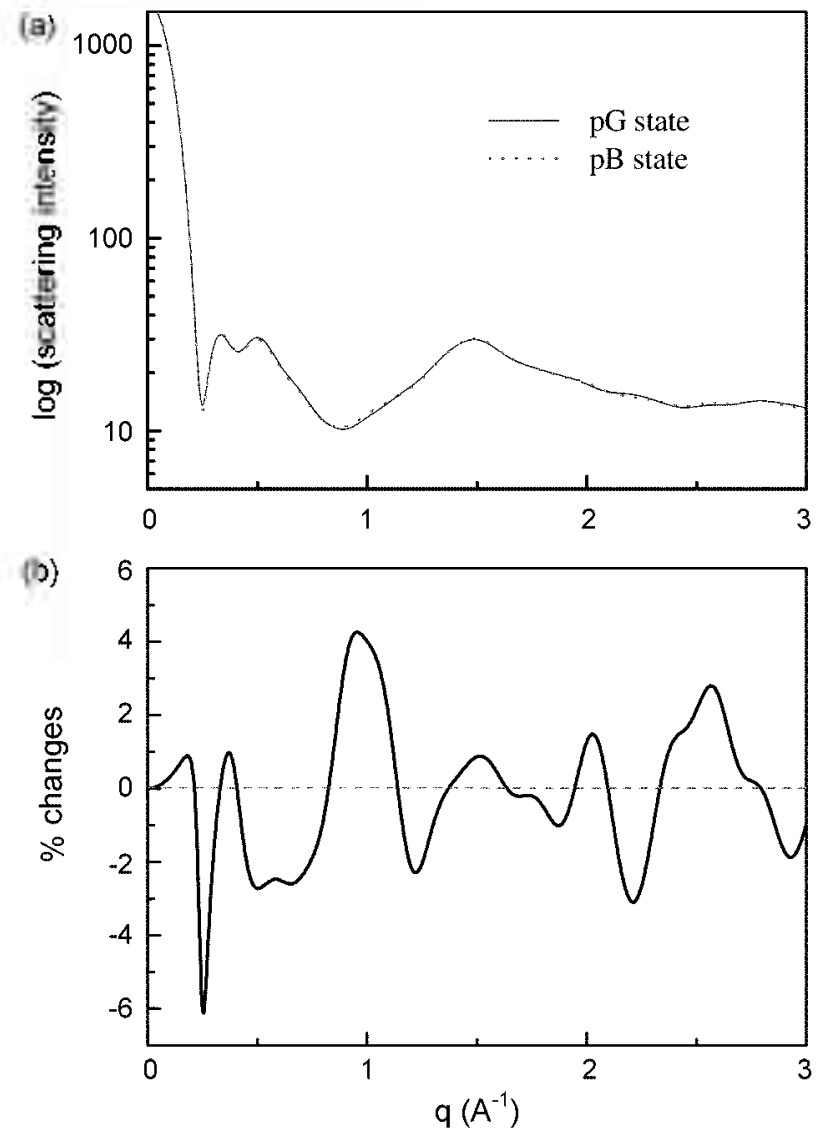

Figure 4. (a) Comparison of calculated scattering pattems for $\mathrm{pG}$ and $\mathrm{pB}$ states of PYP. Scattering pattens tor $\mathrm{pG}$ and $\mathrm{pB}$ states are shown in solid and dotted line, respectively. (b) $\mathrm{pB}-\mathrm{minus}-\mathrm{pG}$ difference scattering pattens calculated from crystallographic atomic coordinates of PYP. 
scattering pattern are roughly assigned according to intramolecular distances of PYP. However. one should aware that this classification is not definite and in principle, all internuclear distances contribute to the overall scattering angles.

Calculation of Light-induced Changes in Wide Angle Scattering. The light-induced change in PYP provides a useful target to probe the sensitivity of wide-angle scattering to the fine structural changes of the protein during its photocycle. Scattering patterns on $\mathrm{pG}$ and $\mathrm{pB}$ states of PYP based on structural models from crystallographic study ${ }^{33}$ were calculated and shown in Figure 4(a). This result suggests that wide-angle scattering patterns should be sensitive to structural difference between $\mathrm{pG}$ and $\mathrm{pB}$ states if they are measured with sufficient precision and resolution. The difference of scattering patterns is shown in Figure 4 (b). plotted as the percentage change in scattered intensity. The difference of scattering patten calculated from the $\mathrm{pG}$ and $\mathrm{pB}$ states of PYP coordinates shows an oscillating structure with maximum anplitude to about $5 \%$ change in scattered intensity. The high X-ray flux from the third generation synchrotron source pernits high precision measurements. For example. the average standard deviation measured with respect to the amplitude of scattering in the region $0.2<q<$ $1.0 \AA^{-1}$ was $0.4 \%$. Therefore, experimental difference scattering patterns in high-angle region can be measured with the sufficient sensitivity to elucidate the statedependent small structural changes of PYP. Work is under progress to measure the time-resolved wide-angle $\mathrm{X}$-ray scattering patterns of PYP using a third generation synchrotron source.

\section{Conclusion}

The static wide-angle X-ray scattering pattern of PYP in solution in the range from $-0.08 \AA^{-1}$ to $-2.8 \AA^{-1}$ was measured using a third generation synchrotron $\mathrm{X}$-ray source. Wide-angle scattering patterns are sensitive to the detailed structures of protein such as domain structures, secondary structures, and their packing. Furthermore these wide-angle scattering patterns give a chance to make a comparison between experimental scattering patterns and detailed structural models determined by the X-ray crystallography. $\mathrm{X}$-ray scattering profiles for the intermediate $(\mathrm{pB}$ state) and ground state $(\mathrm{pG})$ of PYP were calculated. Wide-angle $X$ ray scattering obtained with the high precision should be capable of monitoring light-induced changes in conformation and structural dynamics of PYP.

Acknow ledgment. We are please to dedicate this article to Prof. Yong Hae Kin at KAIST on the occasion of his $65^{\text {th }}$ anniversary. This work was supported by the Office of Basic Energy Sciences. Divisions of Chemical and Material Sciences (Advanced Photon Source). U.S. Department of Energy. under contract W-31-109-ENG-38 to DMT and XZ. and by the Young Scientist Basic Research Program of Korea Science and Engineering Foundation (R08-2004-00010076-0) to HI.

\section{References}

1. Edwards, A. M; Arrowsmith. C. H.: Christendat. D: Dharamsi. A.: Friesent. J. D.: Greenblatt. T. F.: Vedadi. M. Nat. Struct. Biol. 2000. 7,970 .

2. Kim. S.: Jin. G.: Lim. M. Bull Korean Chem Soc. 2003. 24. 1470.

3. Lim. M. Bull. Kowan Chem. Soc. 2002. 23.865.

4. Wulff, M.: Plech, A.: Evbert. L: Randler. R: Schotte. F. Anfinrud. P. Faraday Discussions 2003. 122. 13.

5. Schotte. F.: Lim. M. H.: Jackson, T. A.; Smirnov. A. V.: Soman. J. Olsont. J. S.: Phillips. G. N.: Wulffi. M.: Anfintud. P. A. Science 2003. 300,1944 .

6. Techert, S.; Schotte, F.: Wulti, M. Phys Rev Lett 2001. 86. 2030.

7. Bratos. S.: Mirloup. F.: Vuilleumier. R.: Wultf. M. J. Chem. Plys. 2002. 116,10615

8. Guinier. A. Fournet. G. Small-Angle Scattering of K-rays: John Wiley \& Sons; New: York, 1995.

9. Svergut1. D.: Barberato. C.: Koch. M. H. J. J. Appl. Crust. 1995. 28. 768 .

10. Chacon. P.: Moran. F.; Diaz. J. F; Pantos. E: ANdreu, J. M. Biophus. J. 1998. 74. 2760 .

11. Svergun. D. Biophys. J. $1999,76.2879$.

12. Fischetti. R. F.: Rodi. D. J.: Mirza. A.: Irving. I. C.: Kondrashkina. E.; Makowski. L. J. Sinchrotron Rad 2003. 10. 398.

13. Hirai. M.: Inase. $\mathrm{H} ;$ Havakawa $\mathrm{T} ;$ Mira, $\mathrm{K}$ : Inoue. $\mathrm{K} . J$. Synchoron Rad. 2002. 9.202.

14. Tiede. D. M: Zhang, R:; Seifert, S. Biochemistr 2002, +1. 6605 .

15. Zhang. R: Thiyagarajant. P.: Tiede. D. M. J. Appl. Cryst. 2000. 33.565.

16. Meyer. T. E. Biochent Biophis Acta 1985, 806, 175.

17. Sprenger. W. W.: Hoff. W. D.: Armitage. T. P: Hellingwerf. K. T. J. Bacteriol. 1993. 175.3096.

18. Imamoto. Y; Mihara, K; Tokunaga. F; Kataoka, M. Biochentisty 2001. 40.14336 .

19. Baca. M: Borgstahl. G. E. O.: Boissinot. M: Burke, P. M: Williams. D. R.: Slater. K. A.: Getzoff. E. D. Biochemistry 1994. 33. 14369

20. Borgstahl, G. E. O.: Williams. D. R.: Getzoft. E. D. Biochentisty 1995. 34.6278 .

21. Dux. P.: Rubinstenn, G.: Vuister. G. W. Boelens. R.: Mulder. F. A.: Hard. K: Hoff. W. D.: Kroon. A. R.: Crielaard. W: Hellingwerf, K. J:- Kaptein, R. Biochemistiv 1998, 37.12689.

22. Kort. M.: Vorlk. H.: Xu. X.: Hoff. W. D.: Crielaard. W: Hellingwerf. K. J. FEBS Lett 1996. 382. 73.

23. Imamoto. $Y$; Kataoka. M.; Tokunaga, F.; Asahi. T.: Masuhara. $H$. Biochemisty 2001. 40.6047.

24. U.jj. L.: Devanathan. S.; Mever, T. E.: Cusanovich, M. A.: Tollin. G.: Atkinson. G. H. Biophus. J. 1998. 75. 406 .

25. Hoff, W. D.: Xie. A.: Van Stokkum, I. H. M.; Tang. X.: Gural. J.; Kroon. A. R.: Hellingwerf. K. J. Biochemistiv 1999, 38. 10019

26. Genick. U. K.: Soltis. S. M.: Kuhn1. P.: Canestrelli. I. L.: Getzoff. E. D. Nature 1998. 392, 206.

27. Imamoto. Y.: Ito. T:: Kataoka. M.: Tokunaga. F. FEBS Lett. 1995. $37+157$.

28. Ben1o. M. A.: Jennings. G.: Engbretson. M.: Kn1app. G. S.: Kutz. C.: Zabransky. B.: Linton. J.: Seitert. S.: Wiley. C.: Montano. P. A. Nucl hastrm. Meth. A 2001. 467.690.

29. Warren. B. E. Irav Diffraction: Dover Publication. Inc.: New York. 1990.

30. Fraser. R. D. B.: MacRae. T. P.: Suzuki. E. J. Appl. Civst. 1978. 11. 693 .

31. Hirai. M.: Iwase. H.: Havakawa, T. J. Phs Chent. B 1999. 103.10136.

32. Ducruix. A.: Guilloteau. T. P.: Ries-Kautt. M.: Tardieu. A. J. Cryst. Growth 1996, 168.28.

33. Genick. U. K.: Borgstahl. G. E.: Ng. K.: Ren1. Z.: Pradervand. C. Burke. P. M: Srajer, V: Teng. T. Y.: Schildkamp, W.: McRee, D. E.: Moffät. K.: Getzoff. E. D. Science 1997. 275. 1471.

34. Zhang. R.: Marone. P. A.: Thiyagarajant. P.: Tiede. D. M. Langnuir 1999. 15.7510.

35. Tiede. D. M.: Zhang. R.: Chen. L. X.: Yu. L.: Lindsey. T. S. J. Am. Chent. Soc. 2004, 126. 14054. 